\title{
Quantum electrodynamics near a photonic bandgap
}

\author{
Yanbing Liu and Andrew A. Houck ${ }^{\star}$
}

Photonic crystals are a powerful tool for the manipulation of optical dispersion and density of states, and have thus been used in applications from photon generation to quantum sensing with nitrogen vacancy centres and atoms $\mathrm{s}^{1,2}$. The unique control provided by these media makes them a beautiful, if unexplored, playground for strong-coupling quantum electrodynamics, where a single, highly nonlinear emitter hybridizes with the band structure of the crystal. Here we demonstrate that such a hybridization can create localized cavity modes that live within the photonic bandgap, whose localization and spectral properties we explore in detail. We then demonstrate that the coloured vacuum of the photonic crystal can be employed for efficient dissipative state preparation. This work opens exciting prospects for engineering long-range spin models ${ }^{3,4}$ in the circuit quantum electrodynamics architecture, as well as new opportunities for dissipative quantum state engineering.

The perturbative effect of a structured vacuum is the renowned Purcell effect, which states that the lifetime of an atom in such a space will be proportional to the local photonic density of states (DOS) near the atomic transition frequency. In practice, the birth of the photonic crystal, which greatly modifies the vacuum fluctuations, has enabled the control of spontaneous emission of various emitters, such as quantum $\operatorname{dots}^{5,6}$, magnons ${ }^{7}$ and superconducting qubits ${ }^{8}$. However, when an atom is strongly coupled to a photonic crystal, non-perturbative effects become important, and significantly enrich the physics. For instance, a single-photon bound state has been predicted to emerge within the gap $^{9}$, and spontaneous emission of the atom will thus exhibit Rabi oscillation and light-trapping behaviour. In contrast to electronic bandgap systems, even multiple photons can be simultaneously localized by a single atom, and the coherent photonic transport within the otherwise forbidden bandgap can have a strongly correlated nature ${ }^{10-12}$. In contrast to a system with discrete cavity modes, which is well described by the single-mode or multimode Jaynes-Cummings Hamiltonian ${ }^{13,14}$, a continuous density of states enables the formation of a localized state in the bandgap. While other spin-boson problems with continuous DOS have also been studied experimentally $y^{15,16}$ or theoretically ${ }^{17,18}$ with superconducting circuits, this work explores physics near the band edge, where localized states emerge and reservoir engineering becomes possible.

Light-matter interactions are being actively pursued using cold atoms coupled to optical photonic crystals ${ }^{19,20}$, where the study of photonic band edge effects requires a combination of challenging nanostructure fabrication and optical laser trapping. Although impressive progress has been made, atoms are only weakly coupled to photonic crystal waveguides ${ }^{20}$, potentially limiting the physics to the perturbative regime. In this letter, using a microwave photonic crystal and a superconducting transmon qubit, we are able to reach the strong-coupling regime of quantum electrodynamics near a photonic bandgap. This regime is characterized by the emergence of spectrally resolvable new polariton states, similar to the wellknown vacuum Rabi splitting in cavity quantum electrodynamics. We will give a more quantitative definition of strong coupling in the following discussion.

Our device consists of 14 unit cells, each of which contains two coplanar waveguide (CPW) sections with different lengths $\ell$ and impedances $Z\left(\ell_{\mathrm{lo}}=0.45 \mathrm{~mm}, \ell_{\mathrm{hi}}=8 \mathrm{~mm}\right.$ and $\left.Z_{\mathrm{lo}}=28 \Omega, Z_{\mathrm{hi}}=125 \Omega\right)$. These parameters are chosen so that the band edge is within our measurement window $(4-10 \mathrm{GHz})$ and that the bare photonic crystal has a smooth spectrum. The dispersion relation can be calculated using transfer matrices and is given by

$$
\begin{gathered}
\cos \left(\frac{\omega_{k} \ell_{\mathrm{lo}}}{v_{\mathrm{p}}}\right) \cos \left(\frac{\omega_{k} \ell_{\mathrm{hi}}}{v_{\mathrm{p}}}\right)-\frac{1}{2}\left(\frac{Z_{\mathrm{hi}}}{Z_{\mathrm{lo}}}+\frac{Z_{\mathrm{lo}}}{Z_{\mathrm{hi}}}\right) \sin \left(\frac{\omega_{k} \ell_{\mathrm{lo}}}{v_{\mathrm{p}}}\right) \\
\times \sin \left(\frac{\omega_{k} \ell_{\mathrm{hi}}}{v_{\mathrm{p}}}\right)=\cos \left(k\left(\ell_{\mathrm{lo}}+\ell_{\mathrm{hi}}\right)\right)
\end{gathered}
$$

where $v_{\mathrm{p}}$ is the phase velocity in the waveguide, $\omega_{k}$ is the frequency of the allowed Bloch wave and $k$ is the wavevector in the Brillouin zone. Based on the electric field distribution (Bloch wavefunction), we have purposely placed a transmon qubit in the centre of one unit cell in the middle of the device. This makes the qubit optimally coupled to the second photonic band. Consequently, only this band is taken into account, and a quadratic dispersion relation $E=\hbar \omega_{0}+\alpha\left(k-k_{0}\right)^{2}$ is further assumed. Our device parameters yield $\omega_{0} / 2 \pi=7.7 \mathrm{GHz}$. The complete device image is shown in Fig. 1. It is anchored to the base stage $(15 \mathrm{mK})$ of our dilution refrigerator and connected to a typical $50 \Omega$ measurement chain.

The Hamiltonian of the whole system can be written as

$$
H=\sum_{k} \hbar \omega_{k} a_{k}^{\dagger} a_{k}+\hbar \frac{\omega_{\mathrm{q}}}{2} \sigma^{z}+\sum_{k} \hbar g_{k}\left(a_{k}^{\dagger} \sigma^{-}+a_{k} \sigma^{+}\right)
$$

where $\omega_{\mathrm{q}}, \omega_{k}$ are the frequencies of the bare qubit and the electromagnetic mode with wavevector $k \cdot a_{k}^{\dagger}\left(\sigma^{+}\right)$and $a_{k}\left(\sigma^{-}\right)$are the mode (qubit) raising and lowering operators. We have ignored other dissipation channels of the qubit and have also performed a rotating wave approximation. In the single-particle spectrum, there exists a polariton state within the bandgap with the eigenenergy $\omega_{\mathrm{b}}$ given by the root of the equation

$$
\hbar\left(\omega_{\mathrm{q}}-\omega_{\mathrm{b}}\right) \sqrt{\hbar\left(\omega_{0}-\omega_{\mathrm{b}}\right)}=\pi g^{2} / \alpha
$$

We have already assumed that $g_{k} \approx g$ for all wavevectors $k$, which is valid in our device design. The solution in the bandgap always exists no matter how far the bare qubit frequency $\omega_{\mathrm{q}}$ is detuned from the band edge $\omega_{0}$. In real space, the photonic part of this polariton state is exponentially localized around the qubit (hence the 
a

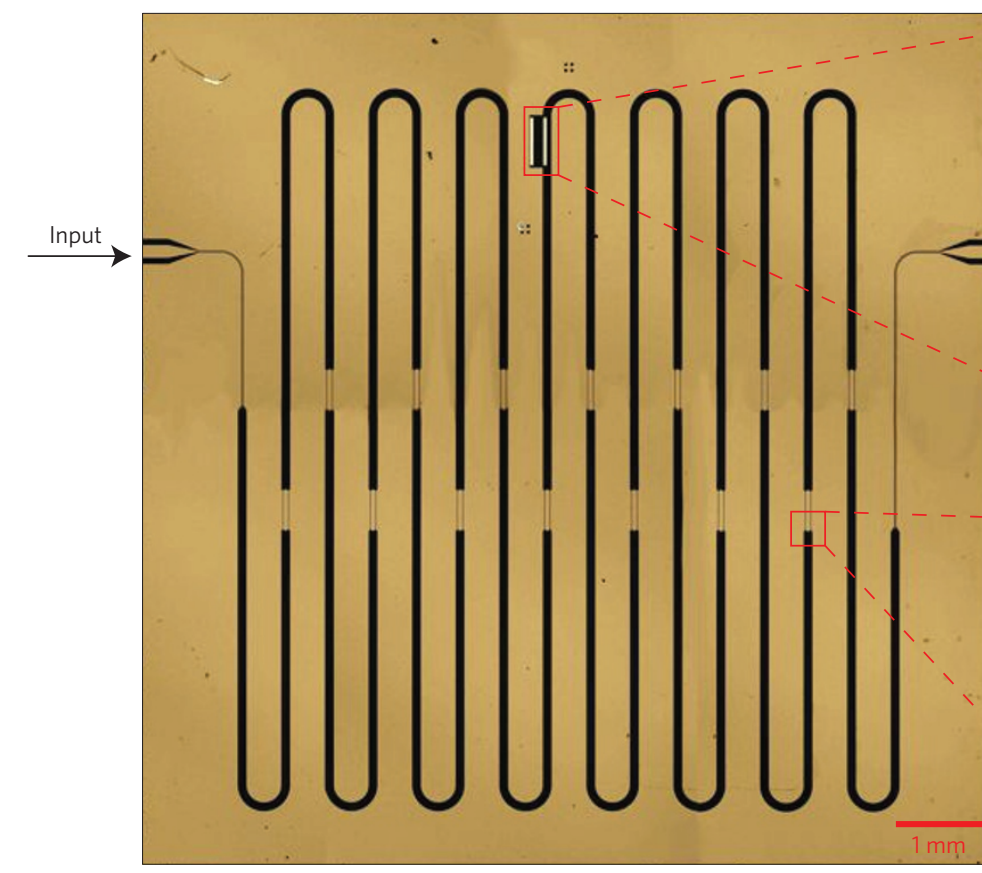

b

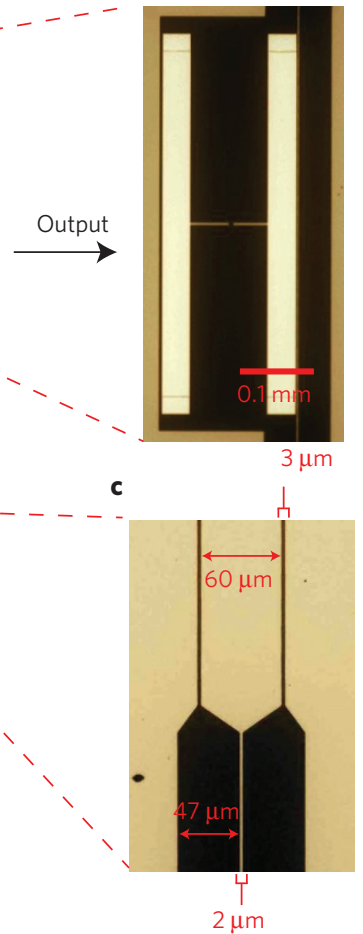

d

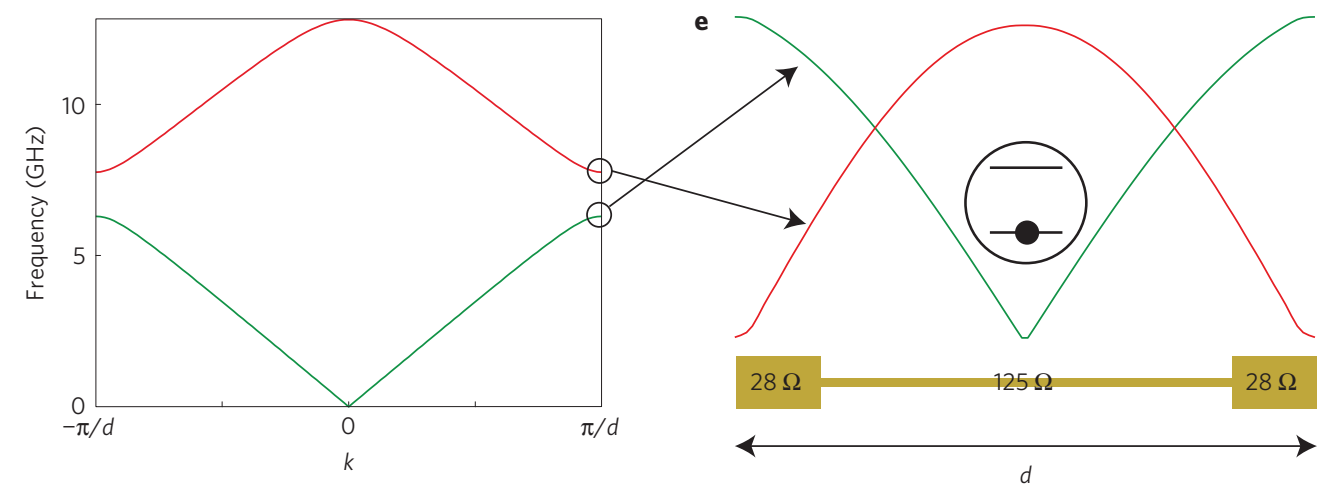

Figure 1 | Device design. a, Optical image of the device. The tan region indicates metals $\mathrm{Nb}$ and $\mathrm{Al}$, while the black region indicates the sapphire substrate. The impedance is periodically modulated by varying the centre pin and gap widths of the CPW. $\mathbf{b}$, A standard transmon qubit made with a split pair of Josephson junctions is coupled to the high-impedance section of the unit cell in the middle of the device. c, High- and low-impedance sections of the waveguide. $\mathbf{d}$, Theoretical band structure of the 1D photonic crystal. e, Schematic of the qubit and the unit cell of the photonic crystal. The green and red curves represent the calculated Bloch wavefunction at $k=\pi / d$ of the first and the second photonic band, respectively. Placing the qubit in the middle of the unit cell maximizes(minimizes) the coupling with the second(first) band.

bound state) with the localization length $L$ given by the penetration depth $L=\sqrt{\alpha / \hbar\left(\omega_{0}-\omega_{\mathrm{b}}\right)}$. The qubit component of this state can be computed to be $P_{\mathrm{q}}=2\left(\omega_{\mathrm{b}}-\omega_{0}\right) /\left(3 \omega_{\mathrm{b}}-\omega_{\mathrm{q}}-2 \omega_{0}\right)$, therefore this state is mostly qubit-like deep within the bandgap, while it is mostly photon-like close to the band edge.

In an infinite photonic crystal, this bound state can result in permanent light trapping ${ }^{12}$ in photonic transport. However, in our finite system, the size of which is comparable to $L$, it is a leaky bound state with a finite spectral linewidth $\gamma$. It can be shown ${ }^{21}$ that $\gamma$ is proportional to the overlap of this state's wavefunction with the externally coupled waveguide $\gamma \sim \mathrm{e}^{-d_{0} / 2 L}$, where $d_{0}$ is the physical length of the device. When probed with a weak signal, this state assists photonic transport within the bandgap; hence, we observe a Lorentzian transmission peak centred at $\omega_{\mathrm{b}}$. As the bare qubit frequency $\omega_{\mathrm{q}}$ is tuned closer to the band edge, the bound state has a larger localization length, and thus carries a larger linewidth.

We measure the bound state linewidth $\gamma$ and exponentially fit the data to the calculated inverse localization length $1 / L$ (Fig. 2b).
This yields the effective device length $d_{\text {fit }}$ to be $140 \pm 12 \mathrm{~mm}$, in agreement with the length of the whole device $d_{0}=126 \mathrm{~mm}$. To further validate the above theoretical model, we focus on the cases where the bare qubit frequency is completely within the band. In Fig. 2a, we observe that the bound state peak below the band edge persists while the input signal at the bare qubit frequency is completely reflected due to destructive interference ${ }^{15}$. Now we can extract $\omega_{\mathrm{q}}, \omega_{\mathrm{b}}$ and fit the data to equation (3). Note that when the bare qubit is resonant with the band edge, the predicted energy shift is $\Delta / 2 \pi=\left(\omega_{\mathrm{b}}-\omega_{0}\right) / 2 \pi=\left(\pi g^{2} / \alpha\right)^{2 / 3}(1 / h)$ and $P_{\mathrm{q}}=2 / 3$. We use $\Delta / 2 \pi$ as the fitting parameter instead of $g$ so that we can then define the strong-coupling regime as $\Delta \gg \kappa$, where $\kappa$ characterizes the steepness of the band edge. In our device, the best fit yields $\Delta / 2 \pi=250 \mathrm{MHz}$, while $\kappa / 2 \pi \approx 26 \mathrm{MHz}$ (see Supplementary Information).

Unlike harmonic defect states, this bound state can be used to control quantum transport within the bandgap. We achieve this by taking advantage of the anharmonic multilevel structure of the 


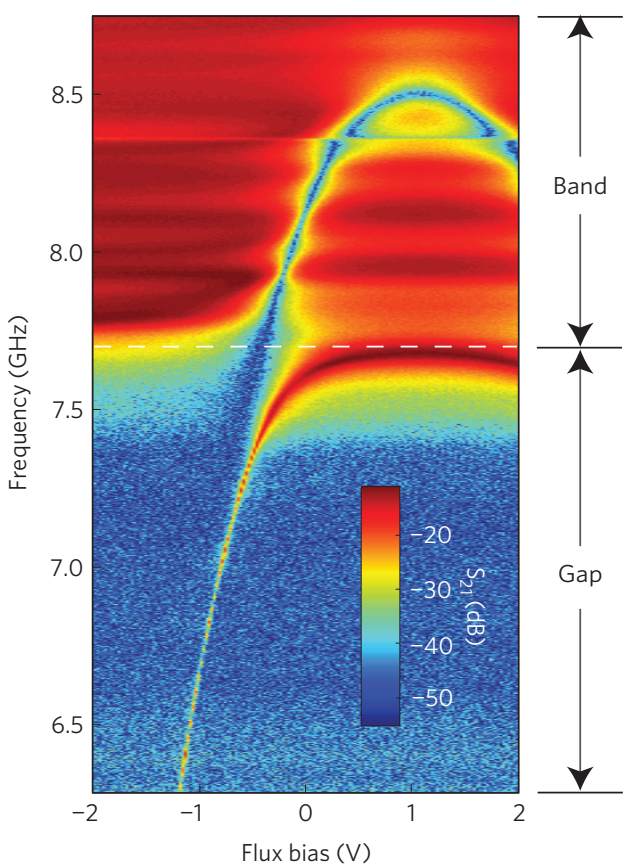

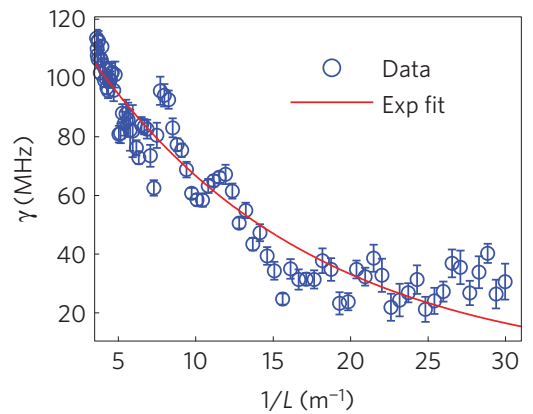

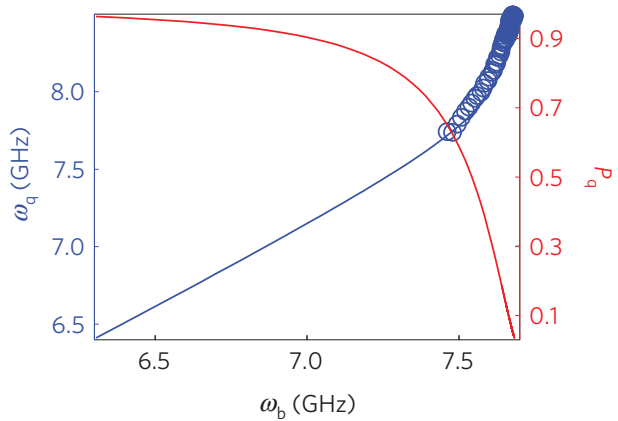

Figure 2 | Single-photon bound state. a, Low-power transmission of the device as the qubit is tuned across the band edge. The colour bar represents the transmission amplitude on a log scale and the dashed line indicates the band edge $\omega_{0}$. The persistent peak in the gap indicates the single-photon bound state. Note that the defect state at $8.36 \mathrm{GHz}$ does not couple to the qubit. $\mathbf{b}$, Relation between the bound state linewidth and the localization length $L$. The error bars of the linewidth data represent the standard deviation of the Lorentzian fit. Based on the theory in the main text, we exponentially fit the linewidth data (see Supplementary Information). c, Relation between the bare qubit frequency and the qubit component with the bound state frequency. The blue curve is extrapolated based on fitting the data when the qubit is within the band.

transmon qubit (anharmonicity $\mathrm{E}_{c} / 2 \pi=385 \mathrm{MHz}$ ). We tune the bound state deep into the gap $\omega_{0}-\omega_{\mathrm{b}} \gg \Delta$, resonantly pump it with Rabi rate $\Omega_{\mathrm{p}}$, and apply another weak tone to probe the transmission. In the limit of $\Omega_{\mathrm{p}} \gg \gamma$, we observe, in Fig. 3a, four extra transmission peaks due to the appearance of Rabi sidebands and an Autler-Townes (AT) splitting of $\omega_{12}$, while the bound state peak is strongly suppressed. The AT splitting arises due to the Rabi splitting of the $|1\rangle \leftrightarrow|2\rangle$ transition. Furthermore, the AT splitting doublet has a much larger transmission amplitude than the Rabi sidebands. Similarly, in Fig. 3b, when the pump tone is resonant with the second transition $\omega_{\mathrm{p}}=\omega_{12}$, we only observe the AT splitting of $\omega_{01}$, also known as electromagnetically induced transparency (EIT) of a single atom. Here the EIT effect is revealed in the suppression of transmission at $\omega_{01}$ within the bandgap.

These observations are attributed to the coupling of the laser-dressed states with the photonic crystal and their different steady-state populations. Essentially, a photon can transmit through the bandgap only if the resonant dressed-state transition $|\nu, N\rangle \leftrightarrow|\mu, N+1\rangle$ is strongly coupled to the waveguide and not population inverted $\left(\rho_{\nu v}>\rho_{\mu \mu}\right)$, where $\rho_{\nu \nu}=\langle\nu|\rho| v\rangle$ and $|\nu, N\rangle$ indicate laser-dressed states that include $N$ laser photons. This result can be arrived at by assuming a linear response and using a transfer matrix technique ${ }^{22}$. In a waveguide with linear dispersion, the resonant transmission coefficient $t_{\mathrm{q}}$ of a dressed-state transition can be simplified $\mathrm{as}^{23}$,

$$
t_{\mathrm{q}}=1+\eta\left(\rho_{\nu \nu}-\rho_{\mu \mu}\right)
$$

where $0<\eta \leq 1$ is a quantity that characterizes the coupling between the dressed-state transition and the waveguide. Combining this with transfer matrices of periodic waveguides allows us to determine the total transmission coefficient $t$ in the bandgap. It is readily apparent that the probe signal is amplified (attenuated) when the population is inverted (not inverted) in a normal waveguide, while the opposite is true within the photonic bandgap. For instance, in Fig. 3a near the bound state frequency $\omega_{01}$, when $\Omega_{\mathrm{p}} \ll \gamma, t_{\mathrm{q}} \approx 0$ and $r_{\mathrm{q}} \approx-1$, yielding $t \approx 1$. While for $\Omega_{\mathrm{p}} \gg \gamma$, the corresponding dressed states are almost equally populated, equivalent to $t_{\mathrm{q}} \approx 1$ and $r_{\mathrm{q}} \approx 0$, resulting in $t \approx 0$. Here $r_{\mathrm{q}}$ is the reflection coefficient of the qubit. These calculations yield good agreement, and show quantum transport within the bandgap can indeed be coherently controlled with an external drive (see Supplementary Information).

Laser-dressed states can even hybridize with the photonic crystal and form doubly dressed states, just as a single-photon bound state is formed when a bare qubit is tuned near the band edge. Although being a archetypal quantum optics model, analytical treatment of resonance fluorescence near the band edge is not available ${ }^{24}$. We present an experimental examination of the driven dynamics as we tune the bound state closer to the band edge. In the pump-probe experiment in Fig. 4a, we observe that when one sideband gets close to the band edge it splits into two resonances, including a peak within the bandgap and a dip within the band. This level splitting ( $\sim 90 \mathrm{MHz})$ is weaker than the direct coupling between the qubit and the photonic crystal $\Delta$ (see Supplementary Information). This spectral information underlies the non-Markovian light emission dynamics; that is, the emitted light at the sideband can be reflected back by the photonic medium and reabsorbed by the qubit.

The deep transmission dip around the upper sideband can be interpreted as dressed-state cooling, which means that the qubit is dynamically pumped into one specific quantum state. Ignoring the band edge effect and higher transmon levels, the reduced dynamics of the qubit can be described by the following master equation (see Supplementary Information)

$$
\begin{gathered}
\frac{\partial \rho}{\partial t}=\frac{\gamma_{o}}{8}\left(\tilde{\sigma}^{z} \rho \tilde{\sigma}^{z}-\tilde{\sigma}^{z} \tilde{\sigma}^{z} \rho\right)+\frac{\gamma_{-}}{8}\left(\tilde{\sigma}^{+} \rho \tilde{\sigma}^{-}-\tilde{\sigma}^{-} \tilde{\sigma}^{+} \rho\right) \\
+\frac{\gamma_{+}}{8}\left(\tilde{\sigma}^{-} \rho \tilde{\sigma}^{+}-\tilde{\sigma}^{+} \tilde{\sigma}^{-} \rho\right)+\text { h.c. }
\end{gathered}
$$


a

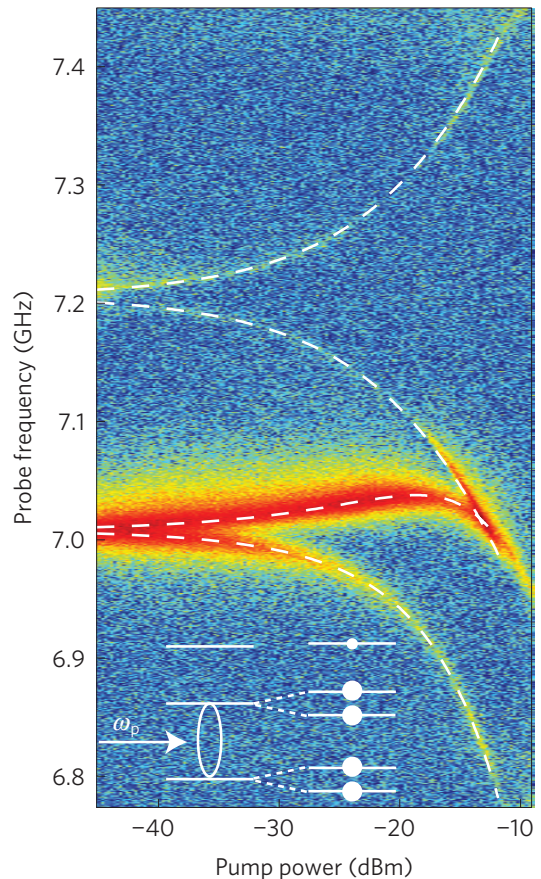

b

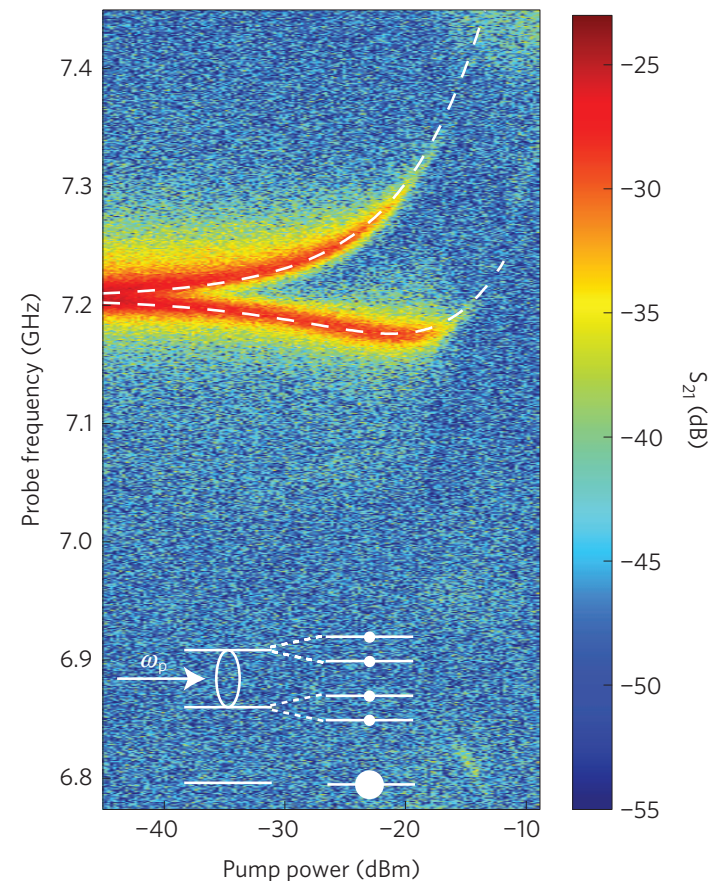

Figure 3 | Pump-probe experiment within the bandgap. The colour bar represents the transmission amplitude of the weak probe signal in log scale. The inset shows the level diagram of the bare states and dressed states. $\mathbf{a}$, The bound state is deep within the bandgap $\left(\omega_{\mathrm{p}}=\omega_{01}=7.206 \mathrm{GHz}\right.$ and $\omega_{12}=7.008 \mathrm{GHz}$ ). The transmission near the bound state frequency $\omega_{01}$ is strongly suppressed, accompanied by two Rabi sidebands at approximately $\omega_{01} \pm \Omega_{\mathrm{p}}$. The most prominent signature is the Autler-Townes splitting of the second transition near $\omega_{12}$. Dashed white lines indicate simulation results. Four transmon states are accounted for in the simulation, where the only fitting parameter is the effective Rabi rate $\Omega_{\mathrm{p}}$. b. $\omega_{\mathrm{p}}=\omega_{12}$. In this case, the AT splitting of $\omega_{01}$ is the dominant feature.

a

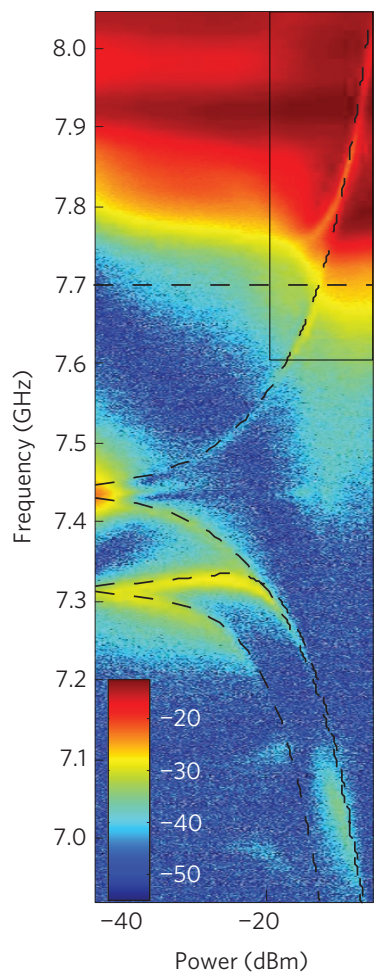

b

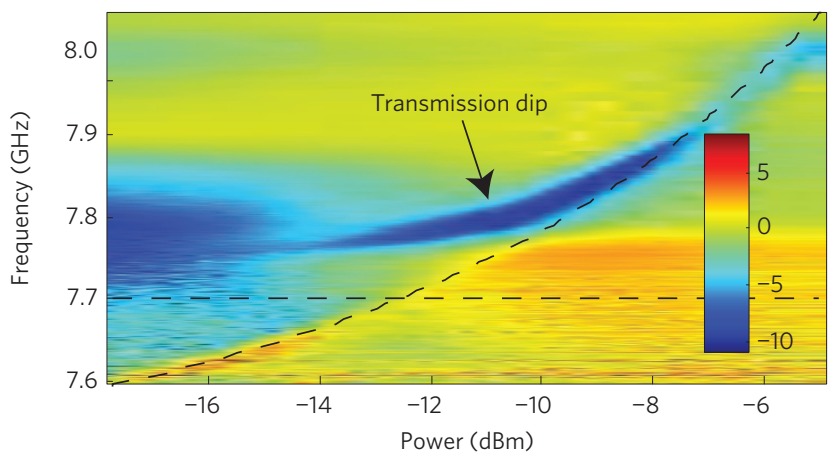

C

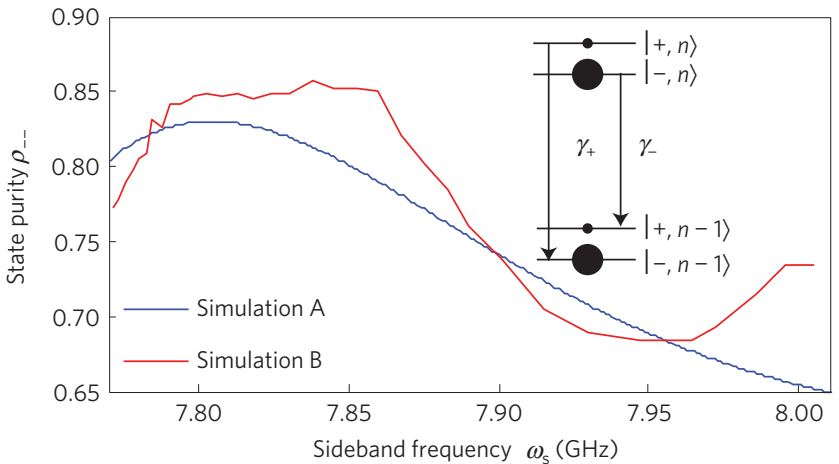

Figure 4 | Pump-probe experiment near the photonic band edge. $\mathbf{a}, \omega_{\mathrm{p}}=\omega_{01}=7.44 \mathrm{GHz}$. Dashed lines indicate the band edge and dressed-state transitions fitted with the same model as in Fig. 3. The sideband that falls within the photonic band in the top-right boxed region is further shown in $\mathbf{b}$. The colour bar represents the transmission amplitude of the weak probe signal on a log scale. $\mathbf{b}$, Zoom-in of the upper sideband. The new colour bar is found by subtracting the bare photonic crystal transmission. Note the appearance of an additional resonance when the sideband is close to the band edge. The resonance within the band is shown in strong attenuation of the probe signal. c, Simulated state purity $\rho_{-}-$using two different methods (see Supplementary Information). The inset shows the schematic of the dressed-state cooling when $\gamma_{+} \gg \gamma_{-}$. 
Here the decay rates of Mollow triplets, $\gamma_{0 \pm}$, are proportional to the local photonic DOS and $\tilde{\sigma}^{z,+,-}$ are Pauli matrices in the dressed state $| \pm\rangle=(1 / \sqrt{2})(|0\rangle \pm|1\rangle)$ basis. It is clear that the steady-state population of the dressed state $|-\rangle$ is $\rho_{--}=\gamma_{+} /\left(\gamma_{-}+\gamma_{+}\right)$. As a result, the qubit will be polarized to the $|-\rangle$ state if the upper sideband falls in the photonic band while the lower sideband falls in the bandgap $\left(\gamma_{+} \gg \gamma_{-}\right)$. Linewidth data in Fig. 2a are used to estimate $\gamma_{ \pm}$in the system. Further taking into account the higher transmon level, we then approximate the dressed-state purity as $\rho_{--} \approx 1-2 \gamma_{-} / \gamma_{+}$(method A). Alternatively, the attenuation of the probe signal can be used to deduce $\rho_{--} \approx 1-t_{\mathrm{q}} / 2$ based on the above linear response theory (method B). Detailed quantitative analysis using these two methods is given in the Supplementary Information and the results are shown in Fig. 4c. Simulated $\rho_{--}$ reaches a maximum of $83 \%$ near the band edge where $\gamma_{+}$is largest.

In comparison with a prior reservoir engineering approach in which a similar effect was demonstrated ${ }^{25}$, here only one external drive is required and the cooling effect is caused by the coloured vacuum of the photonic crystal. This mechanism could be used to stabilize an arbitrary state on the Bloch sphere by detuning the drive from the qubit. Future versions of the device can purposely incorporate a defect cavity mode to assist dispersive readout of the qubit state and incorporate more unit cells to increase the cooling fidelity. Furthermore, this can be directly generalized to the many-qubit case where a dynamical quantum phase transition would be observable and highly entangled many-body states can be stabilized ${ }^{26,27}$. Also, by engineering the coupling of the qubit with multiple bands, a potential wideband dressed-state laser and amplifier can be envisioned.

In the future, low-noise amplifiers can be integrated to study quantum correlation effects in coherent multiphoton transport. The superconducting transmon qubit can be replaced by a flux qubit to reach the ultrastrong-coupling regime ${ }^{28,29}$. The concept can be generalized to three-dimensional architectures and other quantum emitters. Finally, this provides a platform for studying spin models with coupling mediated by overlapping photonics bound states ${ }^{3,4}$, with built-in initialization through reservoir engineering.

\section{Methods}

Methods, including statements of data availability and any associated accession codes and references, are available in the online version of this paper.

Received 22 March 2016; accepted 21 June 2016; published online 1 August 2016

\section{References}

1. Li, L. et al. Coherent spin control of a nanocavity-enhanced qubit in diamond. Nature Commun. 6, 6173 (2015).

2. Clevenson, H. et al. Broadband magnetometry and temperature sensing with a light-trapping diamond waveguide. Nature Phys. 11, 393-397 (2015).

3. Douglas, J. S. et al. Quantum many-body models with cold atoms coupled to photonic crystals. Nature Photon. 9, 326-331 (2015).

4. Gonzalez-Tudela, A., Hung, C.-L., Chang, D. E., Cirac, J. I. \& Kimble, H. J. Subwavelength vacuum lattices and atom-atom interactions in two-dimensional photonic crystals. Nature Photon. 9, 320-325 (2015).

5. Lodahl, P. et al. Controlling the dynamics of spontaneous emission from quantum dots by photonic crystal. Nature 430, 654-657 (2004).

6. Fujita, M., Takahashi, S., Tanaka, Y., Asano, T. \& Noda, S. Simultaneous inhibition and redistribution of spontaneous light emission in photonic crystals. Science 308, 1296-1298 (2005).

7. Hoeppe, U. et al. Direct observation of non-Markovian radiation dynamics in 3D bulk photonic crystals. Phy. Rev. Lett. 108, 043603 (2012).
8. Bronn, N. T. et al. Broadband filters for abatement of spontaneous emission for superconducting qubits. Appl. Phys. Lett. 107, 172601 (2015).

9. John, S. \& Wang, J. Quantum electrodynamics near a photonic band gap: photon bound states and dressed atoms. Phys. Rev. Lett. 64, 2418-2421 (1990).

10. Rupasov, V. I. \& Singh, M. Quantum gap solitons and many-polariton-atom bound states in dispersive medium and photonic band gap. Phys. Rev. Lett. 77, 338-341 (1996).

11. John, S. \& Rupasov, V. I. Multiphoton localization and propagating quantum gap solitons in a frequency gap medium. Phys. Rev. Lett. 79, 821-824 (1997).

12. Longo, P., Schmitteckert, P. \& Busch, K. Few-photon transport in low-dimensional systems: interaction-induced radiation trapping. Phys. Rev. Lett. 104, 023602 (2010).

13. Yoshie, T. et al. Vacuum Rabi splitting with a single quantum dot in a photonic crystal nanocavity. Nature 432, 200-203 (2004)

14. Sundaresan, N. M. et al. Beyond strong coupling in a multimode cavity. Phys. Rev. X 5, 021035 (2015).

15. Astafiev, O. et al. Resonance fluorescence of a single artificial atom. Science 327, 840-843 (2010).

16. Hoi, I.-C. et al. Generation of nonclassical microwave states using an artificial atom in 1D open space. Phys. Rev. Lett. 108, 263601 (2012).

17. Le Hur, K. Kondo resonance of a microwave photon. Phys. Rev. B 85, 140506(R) (2012).

18. Goldstein, M., Devoret, M. H., Houzet, M. \& Glazman, L. I. Inelastic microwave photon scattering off a quantum impurity in a Josephson-junction array. Phys. Rev. Lett. 110, 017002 (2013).

19. Thompson, J. D. et al. Coupling a single trapped atom to a nanoscale optical cavity. Science 340, 1202-1205 (2013).

20. Goban, A. et al. Atom-light interactions in photonic crystal. Nature Commun. 5, 3808 (2014)

21. Biondi, M., Schmidt, S., Blatter, G. \& Tureci, H. E. Self-protected polariton states in photonic quantum metamaterials. Phys. Rev. A 89, 025801 (2014).

22. Shen, J. T. \& Fan, S. Coherent single-photon transport in a one-dimensional waveguide coupled with superconducting quantum bits. Phys. Rev. Lett. 95, 213001 (2005)

23. Koshino, K. et al. Observation of the three-state dressed states in circuit quantum electrodynamics. Phys. Rev. Lett. 110, 263601 (2013).

24. Breuer, H. P. \& Petruccione, F. The Theory of Open Quantum Systems (Oxford Univ. Press, 2007)

25. Murch, K. W. et al. Cavity-assisted quantum bath engineering. Phys. Rev. Lett. 109, 183602 (2012).

26. John, S. \& Quang, T. Quantum optical spin-glass state of impurity two-level atoms in a photonic band gap. Phys. Rev. Lett. 76, 1320-1323 (1996).

27. John, S. \& Quang, T. Collective switching and inversion without fluctuation of two-level atoms in confined photonic systems. Phys. Rev. Lett. 78 1888-1891 (1997)

28. Yoshihara, F. et al. Superconducting qubit-oscillator circuit beyond the ultrastrong-coupling regime. Preprint at http://arXiv.org/abs/1602.00415 (2016).

29. Forn-Díaz, P. et al. Ultrastrong coupling of a single artificial atom to an electromagnetic continuum. Preprint at http://arXiv.org/abs/1602.00416 (2016).

\section{Acknowledgements}

The authors would like to acknowledge D. Sadri, G. Zhang, N. M. Sundaresan and J. Simon for valuable discussions. This work is supported by IARPA under contract W911NF-10-1-0324 and the US National Science Foundation through Materials Research Science and Engineering Centers under contract DMR-1420541.

\section{Author contributions}

Y.L. designed the device, performed the measurements and analysed the data. A.A.H. supervised the whole experiment. All authors contributed to the preparation of this manuscript.

\section{Additional information}

Supplementary information is available in the online version of the paper. Reprints and permissions information is available online at www.nature.com/reprints.

Correspondence and requests for materials should be addressed to A.A.H.

\section{Competing financial interests}

The authors declare no competing financial interests. 


\section{Methods}

The photonic crystal was made using standard optical lithography and dry etching techniques from a $200 \mathrm{~nm} \mathrm{Nb}$ thin film on a $10 \mathrm{~mm} \times 10 \mathrm{~mm}$ sapphire substrate.

The pair of Josephson junctions of the transmon qubit were made using the Dolan bridge technique and evaporated with aluminium. The whole device is packaged in a printed circuit board, wire bonded and anchored at the base plate $(15 \mathrm{mK})$ of our dilution refrigerator. An external solenoid magnet is used to apply a magnetic field across the SQUID loop of the qubit.

Data availability. The data that support the plots within this paper and other findings of this study are available from the corresponding author upon reasonable request. 